\title{
SÍNDROME DE WEBINO POSTQUIRÚRGICO, UNA NUEVA FORMA DE ESTE SÍNDROME
}

\author{
POSTSURGICAL WEBINO, A NEW FORM OF THIS SYNDROME
}

\author{
GONZÁLEZ-MARTÍN-MORO J ${ }^{1}$, GILO F², RUBIO-JERÓNIMO J ${ }^{1}$, TAMÉS-HAYE I ${ }^{1}$
}

\begin{abstract}
RESUMEN
Caso clínico: Una mujer de 20 años de edad fue sometida a resección de un ependimoma del cuarto ventrículo. Después de la cirugía la paciente comenzó a sufrir diplopia y osciloscopia, siendo diagnosticada de síndrome de WEBINO (wall-eyed bilateral internuclear ophthalmoplegia), nistagmus vertical y desviación oblicua (skew).

Discusión: El síndrome de WEBINO es considerado una forma especial de oftalmoplejia internuclear bilateral. Se caracteriza por la ausencia de aducción y nistagmus en abducción en ambos ojos, ausencia de convergencia y con frecuencia exotropia. Hasta el momento se han descrito etiologías isquémicas, desmielinizantes e infecciosas. Sin embargo probablemente este es el primer caso de síndrome de WEBINO de etiología posquirúrgica publicado.
\end{abstract}

Palabras clave: Oftalmoplejía internuclear bilateral, WEBINO, ependimoma.

\begin{abstract}
Case report: A 20 year-old woman was submitted to resection of an ependymome of the fourth ventricle. After surgery the patient began to refer diplopia and oscillopsia. WEBINO syndrome (wall-eyed bilateral internuclear ophthalmoplegia), vertical nystagmus and skew deviation were diagnosed.

Discussion: WEBINO syndrome is considered a special form of bilateral internuclear ophthalmoplegia. It is characterized by bilateral absence of adduction, nystagmic abduction of both eyes, convergence deficiency and frequently exotropia. Ischemic, demyelinating and infectious etiologies have been described, but to our knowledge this is the first report of a postsurgical form of this syndrome (Arch Soc Esp Oftalmol 2009; 84: 407-410).
\end{abstract}

Key words: Wall-eyed bilateral internuclear ophthalmoplegia, WEBINO, ependymome.

\section{CASO CLÍNICO}

Mujer de 20 años sin antecedentes personales de interés que ingresa para intervención de tumoración de fosa posterior adyacente al IV ventrículo. La paciente debutó con hidrocefalia, por lo que previa- mente se había implantado una válvula ventrículoperitoneal para el control de la presión intracraneal. Dos semanas después es sometida a una segunda intervención con la finalidad de resecar el tumor. En el postoperatorio presenta múltiples complicaciones: diplopía binocular, oscilopsia, desviación de la

\footnotetext{
Recibido: 7/7/08. Aceptado: 21/8/09.

Hospital de la Princesa. Madrid. España.

${ }^{1}$ Licenciado en Medicina. Servicio de Oftalmología.

${ }^{2}$ Licenciado en Medicina. Servicio de Neurología.

Presentado como Póster en el Congreso de la International Neuro-Ophthalmology Society (INOS) (Napa, California, 2008).

Correspondencia:

Julio González Martín-Moro

Servicio de Oftalmología

Hospital de la Princesa

C/. Diego de León, 62

28006 Madrid

España

E-mail: juliogmm@yahoo.es
} 
comisura bucal, disfagia y debilidad en las 4 extremidades. En la exploración física se objetivan funciones superiores preservadas, paresia de la aducción de ambos ojos, junto con nistagmus en abducción de ambos ojos (fig. 1), pupilas isocóricas y normorreactivas y tetraparesia $4 / 5$ en brazos y $3 / 5$ en piernas, paresia facial inferior izquierda y desviación de la lengua a la izquierda con atrofia de hemilengua ipsilateral por paresia del hipogloso izquierdo. El estudio anatomopatológico revela que la tumoración es un ependimoma del IV ventrículo grado II de la OMS. En la resonancia magnética craneal postquirúrgica se evidencian restos tumorales con componente hemorrágico en la región del IV ventrículo, así como cambios de señal asociados a restricción de la difusión en regiones frontoparietales bilaterales compatibles con infartos cerebrales de territorios frontera en relación con episodio de bajo gasto (fig. 2). Este daño isquémico cortical en territorios frontera justifica la aparición de la tetraparesia.

A lo largo de estos seis meses, tanto la tetraparesia como el cuadro oftalmológico han evolucionado de forma favorable (fig. 1). En el momento en el que se escribe este artículo ha desparecido la exotropia y mejorado la convergencia, trasformándose de este modo el cuadro en una oftalmoplejia internuclear bilateral convencional.

\section{DISCUSIÓN}

Las alteraciones oculomotoras que presenta la paciente corresponden con un cuadro clínico descri-
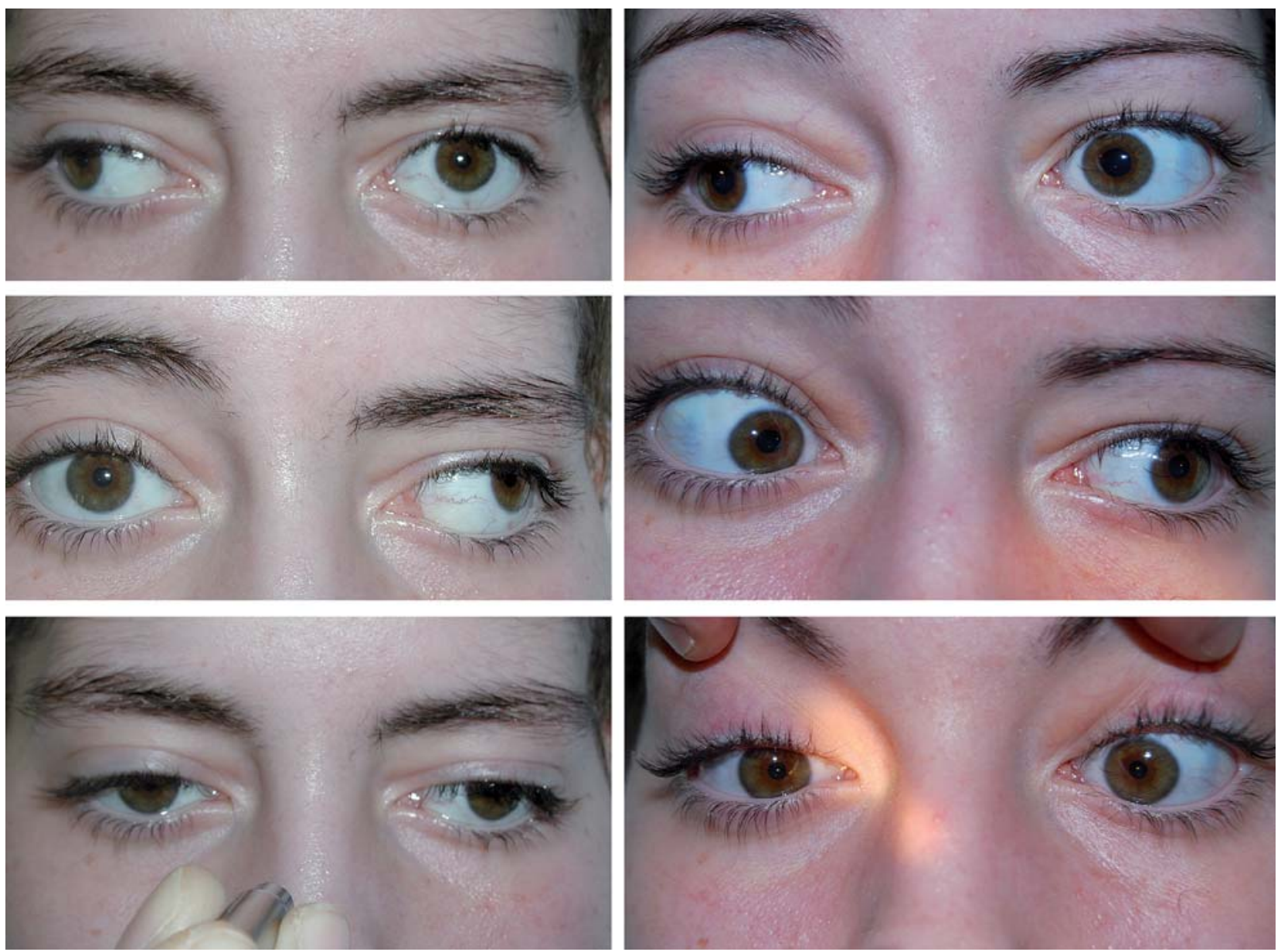

Fig 1: Exploración inicial, tras la cirugía (izquierda), y al cabo de seis meses (derecha). Inicialmente se apreciaba exotropía, limitación bilateral de la aducción e incapacidad para la convergencia. El OI presentaba una desviación oblicua. En seis meses el cuadro evolucionó hasta trasformarse en una oftalmoplejía internuclear «convencional». 
to ocasionalmente en la literatura, y conocido bajo el acrónimo de WEBINO (wall-eyed bilateral internuclear ophthalmoplegia).

Esta forma peculiar de oftalmoplejia internuclear bilateral, cuya patogenia dista de estar clara (1), se caracteriza por la asociación de limitación de la aducción de ambos ojos, acompañada de nistagmus del ojo que abduce, exotropia en posición primaria de la mirada (contrariamente a lo que sucede en los pacientes con oftalmoplejia internuclear, que incluso en los casos bilaterales suelen presentar ortoforia), e incapacidad para la convergencia.

En este caso la paciente también presentaba una hipertropia del OI (desviación oblicua o skew deviation) y nistagmus vertical. Ambas manifestaciones aparecen muy frecuentemente asociadas a la oftalmoplejia internuclear (2). La desviación oblicua tiene además un cierto valor localizador, pues normalmente el ojo hipertrópico es el ojo ipsilateral al fascículo longitudinal medial lesionado (2).

Hasta ahora no se ha publicado una revisión sobre el síndrome y todo lo publicado en referencia a esta misteriosa entidad corresponde a pequeñas series de casos aislados. La etiología coincide con la de la oftalmoplejia internuclear (2), siendo la mayor parte de los casos de causa desmielinizante o isquémica (3). Curiosamente parece existir una cierta influencia étnica, y la mayor parte de los casos publicados en Japón son de causa isquémica, mientras que en los países europeos y Estados Unidos predomina la etiología desmielinizante. También se han descrito algunos casos de causa postraumática (4). Otra etiología referida de forma anecdótica es la infecciosa, en relación con criptococosis (4) o tuberculosis (5).

Desde el punto de vista patogénico, se sabe que la mayor parte de los casos están producidos por lesiones altas en el tronco del encéfalo. Por ello la hipótesis más extendida apunta a que se debe a una lesión combinada del fascículo longitudinal medial y los dos subnúcleos de los rectos medios. Está lesión añadida de los subnúcleos de los rectos medios, explicaría la exotropia frecuentemente asociada y la incapacidad para la convergencia. En este sentido representaría la variante alta del síndrome del uno y medio, en el que se lesionan de forma simultánea el fascículo longitudinal medial y el núcleo del VI par craneal. Sin embargo algunos autores han revisado de forma
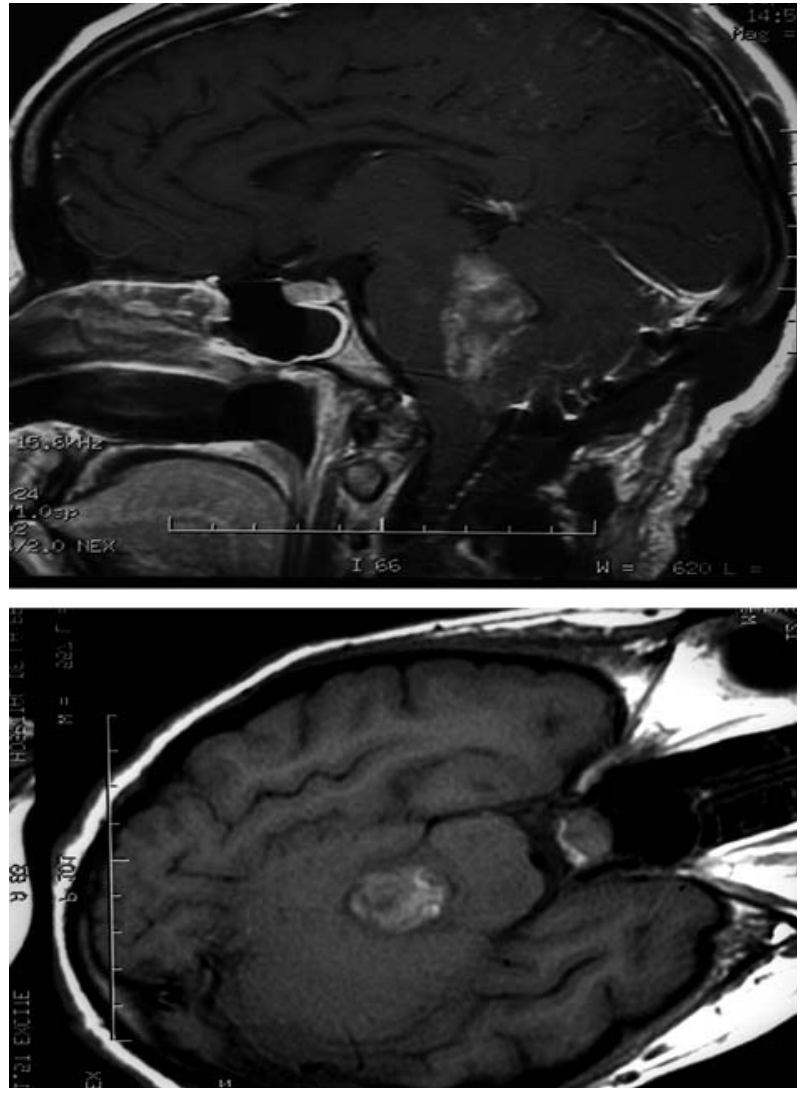

Fig 2: Imagen en la que se aprecia las grandes dimensiones del tumor, y su localización mesencefálica.

minuciosa pequeñas series de este síndrome y llegan a la conclusión de que no necesariamente debe existir afectación de estos subnúcleos (1). La lesión responsable de la insuficiencia de convergencia podría también localizarse a nivel del cerebelo o de la cápsula interna (4).

Dado el mal estado general de la paciente, se desestimó tratamiento con toxina botulínica o cirugía. Tras seis meses de evolución, se ha producido mejoría espontánea, desapareciendo la diplopía y la insuficiencia de convergencia, trasformándose el cuadro clínico en una oftalmoplejia internuclear convencional. En contra de lo que podría esperarse en un principio se ha producido una mejoría parcial del cuadro clínico.

\section{BIBLIOGRAFÍA}

1. Chen CM, Lin SH. Wall-eyed bilateral internuclear ophthalmoplegia from lesions at different levels in the brainstem. J Neuroophthalmol 2007; 27: 9-15. 
2. Smith JW, Cogan DG. Internuclear ophthalmoplegia; a review of fifty-eight cases. Arch Ophthalmol 1959; 61: 687-694.

3. Kim JS, Jeong SH, Oh YM, Soon Yang Y, Kim Y. Teaching NeuroImage: Wall-eyed bilateral internuclear ophthalmoplegia (WEBINO) from midbrain infarction. Neurology 2008; 70: e35.
4. Fay PM, Strominger MB. Wall-eyed bilateral internuclear ophthalmoplegia in central nervous system cryptococcosis. J Neuroophthalmol 1999; 19: 131-135.

5. Inocencio FP, Ballecer R. Tuberculosis granuloma in the midbrain causing wall-eyed bilateral internuclear ophthalmoplegia (Webino). J Clin Neuroophthalmol 1985; 5: 31-53. 